\title{
Absorption Profile of (Poly)Phenolic Compounds after Consumption of Three Food Supplements Containing 36 Different Fruits, Vegetables, and Berries
}

\author{
Letizia Bresciani ${ }^{1}$, Daniela Martini ${ }^{1}$, Pedro Mena ${ }^{1}$, Michele Tassotti ${ }^{1}$, Luca Calani ${ }^{2}$, \\ Giacomo Brigati ${ }^{1}$, Furio Brighenti ${ }^{1}$, Sandra Holasek ${ }^{3}$, Daniela-Eugenia Malliga ${ }^{4}$, \\ Manfred Lamprecht ${ }^{5,6}$ and Daniele Del Rio ${ }^{1,7, *}$ \\ 1 The Laboratory of Phytochemicals in Physiology, Human Nutrition Unit, Department of Food \& Drug, \\ University of Parma, 43125 Parma, Italy; letizia.bresciani@unipr.it (L.B.); daniela.martini@unipr.it (D.M.); \\ pedromiguel.menaparreno@unipr.it (P.M.); michele.tassotti@studenti.unipr.it (M.T.); \\ giacomo.brigati@studenti.unipr.it (G.B.); furio.brighenti@unipr.it (F.B.) \\ 2 Department of Food \& Drug, University of Parma, 43124 Parma, Italy; luca.calani@unipr.it \\ 3 Institute of Pathophysiology and Immunology, Medical University of Graz, A-8010 Graz, Austria; \\ sandra.holasek@medunigraz.at \\ 4 Division of Cardiac Surgery, Department of Surgery, Medical University of Graz, A-8010 Graz, Austria; \\ daniela-eugenia.martin@medunigraz.at \\ 5 Institute of Physiological Chemistry, Medical University of Graz, A-8010 Graz, Austria; \\ manfred.lamprecht@medunigraz.at \\ 6 Green Beat-Institute of Nutrient Research and Sport Nutrition, 8042 Graz, Austria \\ 7 The Need for Nutrition Education/Innovation Programme (NNEdPro), Global Centre for Nutrition and \\ Health, St John's Innovation Centre, Cambridge CB4 0WS, UK \\ * Correspondence: daniele.delrio@unipr.it; Tel.: +39-0521-903-830
}

Received: 30 January 2017; Accepted: 23 February 2017; Published: 26 February 2017

\begin{abstract}
The market of plant-based nutraceuticals and food supplements is continuously growing due to the increased consumer demand. The introduction of new products with relevant nutritional characteristics represents a new way of providing bioactive compounds and (poly)phenols to consumers, becoming a strategy to ideally guarantee the health benefits attributed to plant foodstuffs and allowing the increase of daily bioactive compound intake. A paramount step in the study of nutraceuticals is the evaluation of the bioavailability and metabolism of their putatively active components. Therefore, the aim of the present study was to investigate the absorption profile of the (poly)phenolic compounds contained in three different plant-based food supplements, made of 36 different plant matrices, which were consumed by 20 subjects in an open one-arm study design. Blood samples were collected at baseline and 1,2,5, and $10 \mathrm{~h}$ after capsule intake. Twenty quantifiable metabolites deriving from different (poly)phenolic compounds were identified. Results showed that the consumption of the three capsules allowed the effective absorption of several (poly)phenolic compounds and metabolites appearing at different times in plasma, thereby indicating different absorption profiles. The capsules thus ensured potential health-promoting molecules to be potentially available to target tissues and organs.
\end{abstract}

Keywords: fruit and vegetables; capsules; (poly)phenolic compounds; absorption

\section{Introduction}

Daily consumption of five portions or at least $400 \mathrm{~g}$ of fruits and vegetables is promoted in many national and international dietary guidelines and by public policies [1,2]. An adequate consumption 
of fruit and vegetables has been associated with human health promotion thanks to their role in chronic disease prevention [3]. Conversely, it has been estimated that up to 2635 million deaths per year can be attributable to low fruit and vegetable consumption [4]. In detail, several scientific studies report the correlation between fruit and vegetable consumption with a reduction in all-cause mortality [5], a decrease in overall cancer risk [6], and prevention of metabolic diseases like type II diabetes $[7,8]$. Many national nutrition surveys have shown that the minimum population goal of $400 \mathrm{~g}$ of fruit and vegetables per day established by FAO/WHO is not easily met, especially in selected groups of population such as teenagers $[9,10]$. However, nowadays, consumers are more aware of the health benefits associated with a healthy lifestyle, including high consumption of fruit and vegetables, increasing their will to buy products rich in bioactive compounds [11]. In this context, consumer health demands have boosted the market of plant-based nutraceuticals and food supplements, encouraging the introduction of new products with relevant nutritional characteristics. These products represent new ways of providing bioactive compounds to consumers, becoming a relevant strategy to ideally guarantee the health benefits attributed to plant foodstuffs [12] and allowing the increase of daily bioactive compound intake.

The nutritional relevance of fruit and vegetables on human health could be attributed to their dietary fiber content, mainly soluble, as well as to a large range of micronutrients, including carotenoids, vitamins (mainly vitamin $\mathrm{C}$, and folate), and minerals (as potassium, calcium, and magnesium). In addition, fruit and vegetables are well-recognized sources of non-nutrient bioactive compounds, also called "phytochemicals", among which (poly)phenolic compounds are the predominant and the most investigated [13-17]. The term (poly)phenol includes a number of different chemical structures, including flavonoids and related compounds, but also refers to hydroxycinnamates and phenolic acids, which have only one phenolic ring [18].

A growing amount of experimental research is trying hard to demonstrate the beneficial role of (poly)phenols in humans, trying to unravel the biological mechanisms behind the many proposed effects. Daily consumption of (poly)phenols has been related to reduction of inflammation, hypertension, risk of cardiovascular, metabolic, neurodegenerative diseases, and cancer [18-22]. An essential part of the scientific research in vivo is represented by the investigation of the absorption, metabolism and bioavailability of (poly)phenolic compounds after human intake. In fact, the large structure variety of this class of phytochemicals, together with the food matrices, complex human metabolic pathways and the role of human intestinal microbiota, can deeply influence the metabolism of (poly)phenol compounds, and as a consequence, the availability of their potentially beneficial metabolites to the human body's internal compartments.

The aim of the present study was to investigate the absorption profile of the (poly)phenolic compounds contained in three different plant-based food supplements, made of 36 different vegetal matrices, recently characterized for their (poly)phenol content [23], designed to integrate and increase the daily intake of dietary phenolics. The supplements have been previously shown to exert wide biological effects in diverse health conditions [24-28].

\section{Materials and Methods}

\subsection{Subjects}

The sample size of our study was based on the number of volunteers usually recruited for bioavailability studies involving (poly)phenolic compounds. However, seven subjects would have provided sufficient power ( $\alpha$ error of $0.05,80 \%$ power) to detect a minimum increase of $40 \mathrm{nmol} / \mathrm{L}$ in $2 \mathrm{~h}$ in the absorption kinetics of flavan-3-ols, based on available published data [29]. Twenty participants were recruited from the Graz region (comprising a radius of $50 \mathrm{~km}$ around the center of Graz, Austria) to take part in the study. Selected participants were 9 males (m) and 11 females (f) with the following characteristics: age $34.3 \pm 9.9(\mathrm{~m})$ and $32.9 \pm 9.1$ (f), weight $71.9 \pm 11.7(\mathrm{~m})$ and $63.6 \pm 9.4(\mathrm{f}) \mathrm{kg}$, height $174 \pm 9(\mathrm{~m})$ and $167 \pm 9(\mathrm{f}) \mathrm{cm}$ and BMI 
$23.2 \pm 3.0(\mathrm{~m})$ and $22.8 \pm 3.05(\mathrm{f})\left(\mathrm{kg} / \mathrm{m}^{2}\right)$ (data expressed as mean $\pm \mathrm{SD}$ ). The volunteers had to meet all the inclusion and exclusion criteria. Subjects had to be non-smokers, with a BMI between 20 and $30 \mathrm{~kg} / \mathrm{m}^{2}$, not on medication, not premenopausal, following normal dietary habits (no specific diets, meals and food components.) and adhering to a wash-out period. Exclusion criteria included: not consuming more than four servings of fruits and vegetables per day, not having any type of food allergy or histamine intolerance, not displaying a high level of physical activity (defined as more than five training units/week), not having menstrual dysfunctions and not abusing alcohol.

All participants were informed about the purpose of the study via telephone calls or personal meetings. Subjects who wanted to join the study signed the informed consent form before their inclusion in the trial. Moreover, the selected subjects received a (poly)phenol-poor diet plan which they had to adhere to for $48 \mathrm{~h}$ before the first blood sampling. The list of permitted and forbidden foods is provided in Table S1. Relevant principles of Good Clinical Practice were followed throughout the study. The study was conducted according to the guidelines laid down in the Declaration of Helsinki, and all procedures involving human subjects were approved by the Ethics Committee of the Medical University of Graz, Austria, (EC-number: 27-507ex14/15). The trial was registered at www.clinicaltrials.gov (identifier No. NCT02587468).

\subsection{Test Capsules}

Capsules used for the study were provided by the Juice Plus Company/NSA LLC, Collierville, TN, USA and manufactured for Europe by Natural Alternatives International (NAI), Manno, Switzerland. The capsules contained powdered juice concentrate derived from 36 different fruits, vegetables, and berries including juice and pulp from different vegetal matrices, namely Juice PLUS+ ${ }^{\circledR}$ Vineyard (a berry blend), Juice PLUS $+{ }^{\circledR}$ Fruit Blend and Juice PLUS $+{ }^{\circledR}$ Vegetable Blend, which were kindly supplied by the Juice PLUS $+{ }^{\circledR}$ company. In detail, the powder samples differed for their composition: Juice PLUS $+{ }^{\circledR}$ Vineyard (hereafter called "berry blend") contained $750 \mathrm{mg}$ of dried powder blend of juice and pulp from grapes and berries (45.7\%) including Concord grape, blueberry, cranberry, blackberry, bilberry, raspberry, redcurrant, blackcurrant, elderberry, in varying proportions, besides green tea, ginger root, grape seed, artichoke leaf powder, cocoa powder, and pomegranate powder. Juice PLUS $+{ }^{\circledR}$ Fruit Blend ("fruit blend") instead contained $750 \mathrm{mg}$ of dried powder blend of juice and pulp (52\%) of apple, orange, pineapple, cranberry, peach, acerola cherry, papaya, in varying proportions, beet root, date, and prune. Lastly, Juice PLUS $+{ }^{\circledR}$ Vegetable Blend ("vegetable blend") contained $750 \mathrm{mg}$ of dried powder blend of juice and pulp (60\%) of carrot, parsley, beet, kale, broccoli, cabbage, tomato, and spinach, in varying proportions, as well as sugar beet, garlic powder, oat, and rice bran. Moreover, the fruit and the vegetable powders were enriched with vitamins $C$, and folic acid) and with a natural carotenoid and tocopherol blend. The berry blend powder was enriched with vitamins $C$ and folic acid as well as with a natural tocopherol blend.

\subsection{Study Design}

In an open one-arm study design, three capsules (one berry, fruit, and one vegetable blend), were consumed by each participant on one single occasion. Before the test meal, participants were asked to follow a two-week wash-out period avoiding all food supplements and dietetic products and all kinds of drugs/medications. Additionally, participants were asked to consume a (poly)phenol-poor diet 48 $\mathrm{h}$ before the test day. To facilitate participant adherence to the dietary restrictions, a list of permitted and forbidden foods was supplied. The day of the test, subjects visited the lab after an overnight fast, and after the baseline blood drawing $\left(\mathrm{T}_{0}\right)$, they received the three capsules (one of each blend), to be consumed with $250 \mathrm{~mL}$ of still water. Four additional blood samples were collected, at 1, 2, 5, and $10 \mathrm{~h}$ after capsule intake. After the $2 \mathrm{~h}$ blood sampling, the subjects consumed a standardized (poly)phenol-poor snack, including white bread, cheese, ham, milk, and water ad libitum, in accordance to Pereira-Caro [30]. For each blood drawing, $5 \mathrm{~mL}$ of venous EDTA-blood from the elbow were 
collected via vein catheter, in supine position. Blood was immediately centrifuged at $2500 \mathrm{~g}$ for ten minutes to separate the plasma, which was frozen at $-70{ }^{\circ} \mathrm{C}$ until $\mathrm{uHPLC} / \mathrm{MS}^{\mathrm{n}}$ analyses.

\subsection{Chemicals}

All chemicals and solvents were of analytical grade. 3-Hydroxybenzoic acid, protocatechuic acid, 3-hydroxyphenylpropionic acid, hippuric acid, dihydrocaffeic acid, ferulic acid, dihydroferulic acid, (+)-catechin, 3-caffeoylquinic acid, 5-caffeoylquinic acid, quercetin 3-glucuronide, (hydroxyphenyl)$\gamma$-valeric acid, and pyrogallol were purchased from Sigma-Aldrich (St. Louis, MO, USA). Dihydrocaffeic acid 3-O-sulfate, ferulic acid 4'-O-sulfate, dihydroferulic acid 4'-O-sulfate, caffeic acid 4'-O-glucuronide, dihydrocaffeic acid 3-O-glucuronide, isoferulic acid 3-O-glucuronide were purchased from Toronto Research Chemicals (Toronto, ON, Canada). (3'-hydroxyphenyl)- $\gamma$-valerolactone, (4'-hydroxyphenyl)- $\gamma$-valerolactone, $\left(3^{\prime}, 5^{\prime}\right.$-dihydroxyphenyl)- $\gamma$-valerolactone, ( $3^{\prime}, 4^{\prime}$-dihydroxyphenyl)$\gamma$-valerolactone, $\left(3^{\prime}, 4^{\prime}, 5^{\prime}\right.$-trihydroxyphenyl)- $\gamma$-valerolactone, phenyl- $\gamma$-valerolactone-4'-O-sulfate, phenyl- $\gamma$-valerolactone-3'-O-sulfate, (5'-hydroxyphenyl)- $\gamma$-valerolactone- $3^{\prime}$ - $O$-sulfate, (4'-hydroxyphenyl)$\gamma$-valerolactone- $3^{\prime}$-O-sulfate, phenyl- $\gamma$-valerolactone- $3^{\prime}$-O-glucuronide, phenyl- $\gamma$-valerolactone- $3^{\prime}$, $4^{\prime}$-di-O-sulfate, and (5'-hydroxyphenyl)- $\gamma$-valerolactone- $3^{\prime}$-O-glucuronide were synthesized in house using the synthetic strategy previously outlined by Curti and colleagues [31] and following known procedures reported in the literature [32,33]. Feruloylglycine, 4-hydroxyhippuric acid, quercetin 3'-O-sulfate, hesperetin 3'-O-glucuronide, and hesperetin 7-O-glucuronide were kindly supplied by Professor Alan Crozier. Vanillic acid and (3-methoxy, 4-hydroxyphenyl)acetic acid were purchased from Alfa Aesar (Thermo Fisher (Kandel) $\mathrm{GmbH}$, Postfach, Karlsruhe, Germany) and from Extrasynthese (Genay Cedex, France), respectively. Naringenin 4'-O-glucuronide and naringenin 7-O-glucuronide were purchased from Bertin Pharma (Montigny le Bretonneux, France). Ultrapure water from MilliQsystem (Millipore, Bedford, MA, USA) was used throughout the experiment.

\subsection{Plasma Extraction}

Plasma samples of all volunteers were extracted using a solid phase extraction (SPE) method as reported by Urpi-Sarda and colleagues, with some modifications [34]. Oasis ${ }^{\circledR}$ HLB Vac cartridges (1 cc, $30 \mathrm{mg}$ sorbent, $30 \mu \mathrm{m}$ particle size) (Waters, Milford, Massachusetts, MA, USA) were conditioned with $1 \mathrm{~mL}$ of methanol and equilibrated with $1 \mathrm{~mL}$ of water. An aliquot of $800 \mu \mathrm{L}$ of plasma was added with $16 \mu \mathrm{L}$ of $o$-phosphoric acid (2.5\%) and then loaded into cartridges. The cartridges were washed with $1 \mathrm{~mL}$ of acidified water $(0.1 \%$ formic acid) and finally eluted with $1 \mathrm{~mL}$ of methanol containing formic acid $(1.5 \mathrm{~mol} / \mathrm{L})$. The eluates were evaporated overnight to dryness by means of a rotary speed-vacuum at room temperature and reconstituted with $80 \mu \mathrm{L}$ of methanol/acidified water $\left(0.1 \%\right.$ formic acid) $(50: 50 \mathrm{v} / \mathrm{v})$ prior $\mathrm{uHPLC} / \mathrm{MS}^{\mathrm{n}}$ analysis.

\subsection{UHPLC/MS Analysis}

Plasma extracts were analyzed by a UHPLC DIONEX Ultimate 3000 equipped with a triple quadrupole TSQ Vantage (Thermo Fisher Scientific Inc., San Josè, CA, USA) fitted with a heated-ESI (H-ESI) (Thermo Fisher Scientific Inc., San Josè, CA, USA) probe. Separations were carried out by means of a Kinetex EVO C18 $(100 \times 2.1 \mathrm{~mm})$ column, $1.7 \mu \mathrm{m}$ particle size (Phenomenex, Torrance, CA, USA).

For UHPLC, mobile phase A was acetonitrile containing $0.2 \%$ formic acid and mobile phase $\mathrm{B}$ was $0.2 \%$ formic acid in water. The gradient started with $5 \% \mathrm{~A}$, isocratic conditions were maintained for $0.5 \mathrm{~min}$, and reached $95 \% \mathrm{~A}$ after $6.5 \mathrm{~min}$, followed by $1 \mathrm{~min}$ at $95 \%$. The starting gradient was then immediately reestablished and maintained for $4 \mathrm{~min}$ to re-equilibrate the column. The flow rate was $0.4 \mathrm{~mL} / \mathrm{min}$, the injection volume was $5 \mu \mathrm{L}$, and the column temperature was set at $40^{\circ} \mathrm{C}$.

The applied mass spectrometry (MS) method consisted in the selective determination of each target precursor ion by the acquisition of characteristic product ions in selective reaction monitoring (SRM) mode (Table 1), applying a negative ionization. 
Table 1. Spectrometric characteristics of the 92 monitored compounds, and standard compounds used for quantification of the 20 identified metabolites. Legend: SRM: selective reaction monitoring; ND: not detected.

\begin{tabular}{|c|c|c|c|c|}
\hline Compound & {$[\mathrm{M}-\mathrm{H}]^{-}$} & SRM Transition & S-Lens Value & Quantification \\
\hline Catechol & 109 & 108,81 & 68 & ND \\
\hline Methylcatechol & 123 & 108,81 & 68 & ND \\
\hline Pyrogallol & 125 & $124,81,97$ & 68 & ND \\
\hline Hydroxybenzoic acid & 137 & $91,93,45$ & 70 & ND \\
\hline Hydroxyphenylacetic acid & 151 & 107 & 51 & ND \\
\hline Dihydroxybenzoic acid & 153 & 108,109 & 64 & ND \\
\hline 3-(3'-Hydroxyphenyl)propionic acid & 165 & 119,121 & 48 & ND \\
\hline 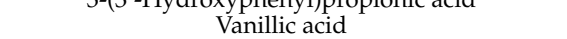 & 167 & $152,108,123$ & 60 & ND \\
\hline Gallic acid & 169 & 125 & 68 & ND \\
\hline Hippuric acid & 178 & 134 & $\begin{array}{l}00 \\
61\end{array}$ & Hippuric acid \\
\hline (3'-Methoxy, 4'-hydroxyphenyl)acetic acid & 181 & 137 & 64 & ND \\
\hline $\begin{array}{l}\text { Dihydrocaffeic acid } \\
\text { Dihetric acia }\end{array}$ & 181 & 137,119 & $\begin{array}{l}04 \\
64\end{array}$ & ND \\
\hline Methylgallic acid & 183 & 168,139 & 70 & ND \\
\hline $\begin{array}{l}\text { Methylganlic accid } \\
\text { Catechol sulfate }\end{array}$ & 189 & $\begin{array}{l}168,139 \\
109,81\end{array}$ & 70 & ND \\
\hline (3'-Hydroxyphenyl)- $\gamma$-valerolactone & 191 & 147,106 & 70 & ND \\
\hline (4'-Hydroxyphenyl)- $\gamma$-valerolactone & 191 & $147,173,103,107$ & 70 & ND \\
\hline Ferulic acid & 193 & 134,178 & 71 & ND \\
\hline (Hydroxyphenyl) $-\gamma$-valeric acid & 193 & $147,149,157,175$ & 72 & ND \\
\hline $\begin{array}{l}\text { 4-Hydroxyhippuric acid } \\
\text { - }\end{array}$ & 194 & 100,150 & 72 & 4-Hydroxyhippuric acid \\
\hline Dihydroferulic acid & 195 & 136 & 73 & ND \\
\hline Syringic acid & 197 & 153,182 & 70 & ND \\
\hline Methylcatechol sulfate & 203 & $123,108,81$ & 70 & ND \\
\hline Pyrogallol sulfate & 205 & $\begin{array}{l}125,124,81,97 \\
125,101\end{array}$ & 68 & Dihydrocaffeic acid 3-O-sulfate \\
\hline (3',5'-Dihydroxyphenyl) $-\gamma$-valerolactone & 207 & $163,123,121$ & 75 & ND \\
\hline (3, $3^{\prime} 4^{\prime}$-Dihydroxyphenyl)- $\gamma$-valerolactone & 207 & $\begin{array}{l}103,12,121 \\
163,122\end{array}$ & 75 & $\mathrm{ND}$ \\
\hline$\left(3^{\prime}, 5^{\prime}\right.$-Dihydroxyphenyl)- $-\gamma$-valeric acid & 209 & $101,124,147$ & 63 & ND \\
\hline$\left(3^{\prime}, 4^{\prime}\right.$-Dihydroxyphenyl)- $\gamma$-valeric acid & 209 & $151,165,191,194$ & 63 & ND \\
\hline $\begin{array}{l}\text { Hydroxybenzoic acid sulfate } \\
\text { Heit }\end{array}$ & 217 & $137,93,45$ & 70 & $\mathrm{ND}$ \\
\hline Methyl-trihydroxybenzoic acid sulfate & 219 & $139,124,125,81,97$ & 68 & Dihydroferulic acid 4'-O-sulfate \\
\hline$\left(3^{\prime}, 4^{\prime}, 5^{\prime}\right.$-Trihydroxyphenyl)- $\gamma$-valerolactone & 223 & $179,205,138$ & 75 & ND \\
\hline Dihydroxybenzoic acid sulfate & 233 & $153,108,109$ & 64 & Dihydrocaffeic acid 3-O-sulfate \\
\hline Hydroxyphenylpropionic acid sulfate & 245 & $165,121,119$ & $\begin{array}{l}04 \\
90\end{array}$ & Dihydrocaffeic acid 3-O-sulfate \\
\hline $\begin{array}{l}\text { My aroxypnenillic acid sulfate } \\
\text { Vanille }\end{array}$ & 247 & $167,152,108,123$ & 90 & 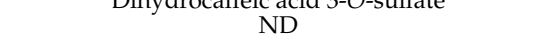 \\
\hline $\begin{array}{l}\text { Gallic acid sulfate } \\
\text { Gate }\end{array}$ & 249 & $16 /, 169,125,123$ & 68 & ND \\
\hline $\begin{array}{l}\text { Gallic accid sulfate } \\
\text { Feruloylglycine }\end{array}$ & 250 & $206,134,162,191,177$ & $\begin{array}{l}68 \\
79\end{array}$ & Feruloylglycine \\
\hline Dihydrocaffeic acid sulfate & 261 & 181,137 & 96 & ND \\
\hline Methylgallic acid sulfate & 263 & $183,168,125$ & 68 & ND \\
\hline Phenyl- $\gamma$-valerolactone $-4^{\prime}-O$-sulfate & 271 & 191,147 & 93 & ND \\
\hline Phenyl- $\gamma$-valerolactone- $3^{\prime}-O$-sulfate & 271 & $191,147,93,80,106$ & 93 & Phenyl- $\gamma$-valerolactone- $3^{\prime}$-O-sulfate \\
\hline $\begin{array}{l}\text { Ferulic acid sulfate } \\
\text { surce }\end{array}$ & 273 & $193,134,178$ & 92 & ND \\
\hline Phenyl- $\gamma$-valeric acid-O-sulfate & 273 & $193,175,157,149,147$ & 92 & ND \\
\hline $\begin{array}{l}\text { Dihydroferulic acid sulfate } \\
\text { Dihe }\end{array}$ & 275 & 195,136 & 75 & ND \\
\hline (5'-Hydroxyphenyl)- $\gamma$-valerolactone- $3^{\prime}$-O-sulfate & 287 & $207,122,163$ & 96 & ND \\
\hline (4'-hydroxyphenyl)- $\gamma$-valerolactone- $3^{\prime}-O$-sulfate & 287 & $207,109,163$ & 96 & (4'-Hydroxyphenyl)- $\gamma$-valerolactone- $3^{\prime}$-O-sulfate \\
\hline 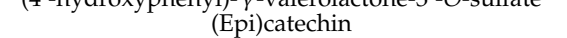 & 289 & $245,203,204.9$ & 98 & 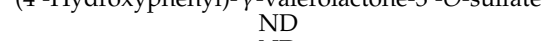 \\
\hline (Hydroxyphenyl)- $\gamma$-valeric acid-O-sulfate & 289 & $209,191,151,147,124,101$ & 92 & ND \\
\hline $\begin{array}{l}\text { Dihydroxyphenyl- } \gamma \text {-valerolactone-O-sulfate } \\
\text { Dihy }-0 \text { - }\end{array}$ & 303 & $209,191,179,223$ & 90 & ND \\
\hline 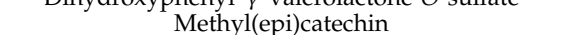 & 303 & $288,245,205$ & 98 & ND \\
\hline Hydroxybenzoic acid glucuronide & 313 & $137,93,45$ & 70 & ND \\
\hline (Methyl-hydroxyphenyl)- $\gamma$-valerolactone-O-sulfate & 317 & 222,237 & 92 & ND \\
\hline Dihydroxybenzoic acid glucuronide & 329 & $153,108,109$ & 64 & ND \\
\hline
\end{tabular}


Table 1. Cont.

\begin{tabular}{|c|c|c|c|c|}
\hline Compound & {$[\mathbf{M}-\mathbf{H}]^{-}$} & SRM Transition & S-Lens Value & Quantification \\
\hline Gallic acid glucuronide & 345 & 169,125 & 68 & ND \\
\hline Apigenin sulfate & 349 & 269,225 & 98 & ND \\
\hline Naringenin sulfate & 351 & 271,151 & 84 & ND \\
\hline 3-Caffeoylquinic acid & 353 & $191,179,135$ & $\begin{array}{l}84 \\
85\end{array}$ & ND \\
\hline 5-Caffeoylquinic acid & 353 & 191 & 85 & ND \\
\hline $\begin{array}{l}\text { 5-Caatfeyylquinic acia } \\
\text { Caffeic acid glucuronide }\end{array}$ & 355 & 179,135 & $\begin{array}{l}85 \\
87\end{array}$ & ND \\
\hline Dihydrocaffeic acid glucuronide & 357 & $181,137,113$ & 63 & ND \\
\hline $\begin{array}{l}\text { Kaempferol sulfate } \\
\text { Kall }\end{array}$ & 365 & 285,257 & 90 & ND \\
\hline Phenyl- $\gamma$-valerolactone- $3^{\prime}$-O-glucuronide & 367 & $191,113,207$ & 93 & ND \\
\hline Phenyl- $\gamma$-valerolactone- $3^{\prime}, 4^{\prime}$-di-O-sulfate & 367 & 287,147 & 93 & $\mathrm{ND}$ \\
\hline Ferulic acid glucuronide & 369 & $193,178,175$ & 92 & Isoferulic acid $3^{\prime}$-O-glucuronide \\
\hline (Epi)catechin sulfate & 369 & $289,245,203,205$ & 98 & ND \\
\hline Dihydroxyphenyl- $\gamma$-valeric acid disulfate & 369 & $209,191,151,147,124$ & 92 & ND \\
\hline $\begin{array}{l}\text { Diosmetin sulfate } \\
\text { Dintain }\end{array}$ & 379 & 299,284 & 90 & Quercetin $3^{\prime}$-O-sulfate \\
\hline Ouercetin $3^{\prime}$-sulfate & 381 & $301,151,179$ & 83 & Ouercetin $3^{\prime}$-O-sulfate \\
\hline Hesperetin sulfate & 381 & $301,151,179$ & 115 & Quercetin $3^{\prime}$-O-sulfate \\
\hline (4'-hydroxyphenyl)- $\gamma$-valerolactone- $3^{\prime}$-O-glucuronide & 383 & 207,163 & 87 & (5'-hydroxyphenyl)- $\gamma$-valerolactone- $3^{\prime}$-O-glucuronide \\
\hline $\begin{array}{l}\text { Methl(epi)catechin sulfate } \\
\text { Mether }\end{array}$ & 383 & $303,288,245,205$ & 98 & ND \\
\hline (Epi)gallocatechin sulfate & 385 & $305,179,221$ & 98 & ND \\
\hline Myricetin sulfate & 397 & $317,316,179$ & 90 & ND \\
\hline Dihydroxyphenyl- $\gamma$-valerolactone-O-glucuronide & 399 & $223,175,179$ & 87 & ND \\
\hline Methyl(epi)gallocatechin sulfate & 399 & $319,304,179,221$ & 98 & ND \\
\hline $\begin{array}{l}\text { Patuletin sulfate } \\
\text { Pate }\end{array}$ & 411 & $331,316,209$ & 90 & Quercetin $3^{\prime}$-O-sulfate \\
\hline Spinacetin sulfate & 425 & 345,330 & 90 & ND \\
\hline Apigenin glucuronide & 445 & 269,225 & 90 & $\mathrm{ND}$ \\
\hline Naringenin $4^{\prime}$-glucuronide & 447 & $271151,379,119$ & 112 & Naringenin $4^{\prime}$-O-glucuronide \\
\hline $\begin{array}{l}\text { Narngenin 4-giucuronice } \\
\text { Naringenin 7-glucuronide }\end{array}$ & 447 & 271,151 & 84 & NDaringenin $4-\mathrm{U}$-giucuroniae \\
\hline Kaempferol glucuronide & 461 & 285,257 & 90 & Quercetin 3-O-glucuronide \\
\hline Phenyl- $\gamma$-valerolactone-- $3^{\prime}, 4^{\prime}-O$-sulfate-O-glucuronide & 463 & $163,207,287,383$ & 87 & ND \\
\hline (Epi)catechin glucuronide & 465 & $289,245,205$ & 98 & ND \\
\hline Diosmetin glucuronide & 475 & 299,284 & 90 & ND \\
\hline Quercetin 3-glucuronide & 477 & $301,151,179$ & 91 & Quercetin 3-O-glucuronide \\
\hline Hesperetin $3^{\prime}$-glucuronide & 477 & 301,113 & 115 & ND \\
\hline Hesperetin 7-glucuronide & 477 & 301,151 & 115 & Hesperetin 7-O-glucuronide \\
\hline Methyl(epi)catechin glucuronide & 479 & $303,288,245,205$ & 98 & ND \\
\hline Hydroxyphenyl- $\gamma$-valerolactone-O-sulfate- $O$-glucuronide & 479 & $303,223,175,259$ & 91 & ND \\
\hline $\begin{array}{l}\text { (Epi)gallocatechin glucuronide } \\
\text { (E) }\end{array}$ & 481 & $305,179,221$ & 98 & ND \\
\hline Myricetin glucuronide & 493 & $317,316,209$ & 90 & Quercetin 3-O-glucuronide \\
\hline Methyl(epi)gallocatechin glucuronide & 495 & $319,304,179,221$ & 98 & ND \\
\hline $\begin{array}{l}\text { Patuletin glucuronide } \\
\text { Pall }\end{array}$ & 507 & $331,316,209$ & 90 & ND \\
\hline Spinacetin glucuronide & 521 & 345,330 & 90 & ND \\
\hline
\end{tabular}


To optimize the method, all the available standard compounds were infused into the MS to set the best mass parameters and to check the actual fragmentation patterns. Finally, for all the analyses, the spray voltage was set at $3 \mathrm{kV}$, the vaporizer temperature at $300^{\circ} \mathrm{C}$, and the capillary temperature operated at $270{ }^{\circ} \mathrm{C}$. The sheath gas flow was 60 units, and auxiliary gas pressure was set to 10 units. Ultrahigh purity argon gas was used for collision-induced dissociation (CID). The S-lens values were defined for each compound based on infusion parameter optimization (Table 1). Conversely, for compounds that were not available for infusion, the S-lens values were set using the values obtained for the chemically closest available standards. Quantification was performed with calibration curves of standards, when available (Table 1). Data processing was performed using Xcalibur software (Thermo Scientific Inc., Waltham, MA, USA). All data were expressed as mean values \pm SEM.

\section{Results}

\section{(Poly)Phenolic Compound Absorption}

The capsules consumed in the present study were previously characterized for (poly)phenolic profile and quantified for their total phenolic content [23]. Capsules were made of 36 different plant matrices, and contained a wide array of different phenolic compounds, principally ellagitannins, flavan-3-ols, flavonols, and anthocyanins in berry blend capsules, flavones, flavonols, flavanones, and anthocyanins in fruit blend capsules, and flavones and flavonols in vegetable blend capsules.

Out of the 92 monitored molecules, 20 quantifiable metabolites were identified, or tentatively identified in plasma samples. All the quantified metabolites were found as conjugated compounds with sulfate, glucuronide or glycine moieties. Three glycine-conjugated metabolites, hippuric acid, 4-hydroxyhippuric acid, and feruloylglycine, were detected. A total of five metabolites were flavonol derivatives, including conjugates of quercetin, kaempferol, myricetin, and patuletin. Three metabolites were directly linked to flavanone metabolism, namely naringenin, and hesperetin derivatives, whereas only one flavone metabolite, namely diosmetin sulfate, was detected. Among flavan-3-ol derivatives, three conjugated phenyl- $\gamma$-valerolactones were detected. Finally, five metabolites were small phenolic derivatives, including hydroxyphenylpropionic acid sulfate, ferulic acid glucuronide, pyrogallol sulfate, dihydroxybenzoic acid sulfate, and methyl-trihydroxybenzoic acid sulfate. Generally, with the exception of hippuric acid, plasma levels of the quantified metabolites did not exceed the nanomolar range. Considering those metabolites for which the origin is strictly attributable to (poly)phenolic compounds contained in the capsules, the most abundant metabolites resulted in the low molecular weight phenolics which are usually produced in the colon by microbial transformation of flavonoids.

The glycine-conjugated metabolites are ubiquitous and could originate both by endogenous precursors [18,35] and by microbial metabolism of phenolic compounds [36-38]. Their potential endogenous origin justifies the high concentration at baseline (Figure S1).

Actually, the absorption curves of hippuric acid and 4-hydroxyhippuric acid did not show a notable concentration peak and their levels were basically constant during the study period. On the contrary, feruloylglycine exhibited a peak plasma concentration $2 \mathrm{~h}$ after consumption, indicating a stronger connection with the phenolic compounds introduced through the capsules, as only the capsules were consumed within the $2 \mathrm{~h}$.

Looking to the other circulating metabolites appearing after capsule consumption, two metabolic phases could be easily distinguished. Conjugated metabolites appearing in the circulatory system within 1 or $2 \mathrm{~h}$ after capsule ingestion suggest an absorption in the first part of the gastro-intestinal tract. Native compounds are rapidly hydrolysed to release the aglycones, which are then conjugated by sulfotransferases (SULTs) and uridine-5'-diphosphate glucuronosyltransferases (UGTs) at both enterocyte and hepatic level before entering the systemic circulatory system [18]. Kaempferol glucuronide, quercetin glucuronide, quercetin sulfate, myricetin glucuronide, and diosmetin sulfate absorption curves are the expression of (poly)phenol metabolism in the first gastro-intestinal tract (Figure 1). 
A

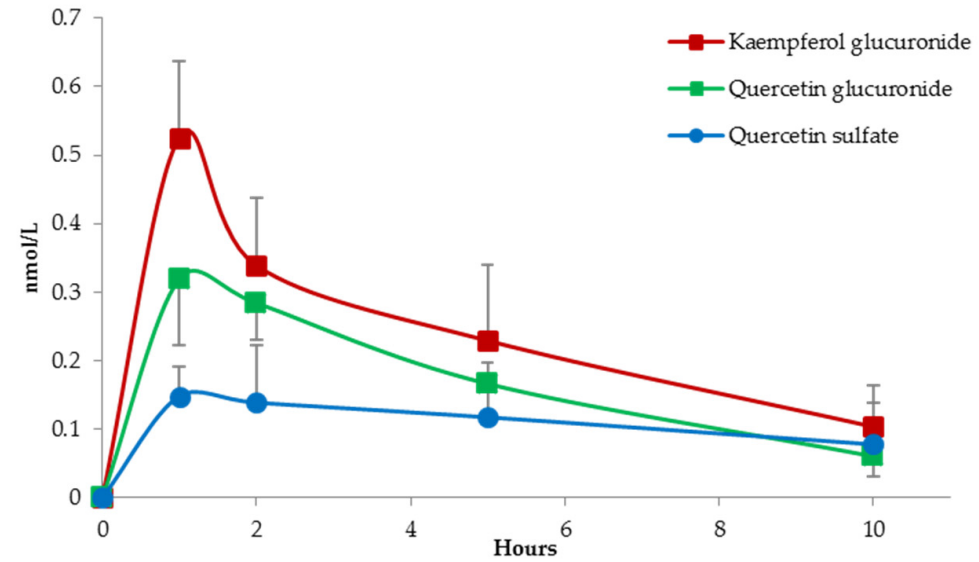

B

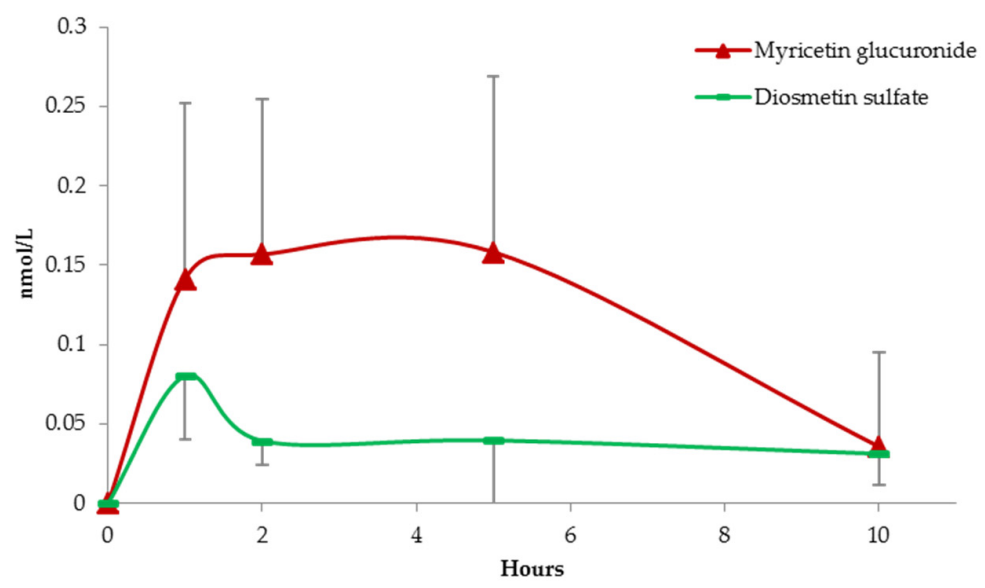

Figure 1. Absorption curves of kaempferol glucuronide, quercetin glucuronide, quercetin sulfate in graph (A); and myricetin glucuronide and diosmetin sulfate in graph (B). Data are expressed as mean values and bars represent standard error of means (SEM).

A concentration peak recorded between 5 and $10 \mathrm{~h}$ after test meal indicates, instead, a clear interaction between the indigested (poly)phenolic fraction and the colonic microbiota. The absorption profile outlined by patuletin sulfate and hesperetin sulfate likely represents a colonocyte level absorption and a subsequent conjugation at hepatic level (Figure 2). Actually, their plasmatic concentration reached a maximum level $5 \mathrm{~h}$ post capsule consumption.

Two quantified metabolites, namely naringenin glucuronide and hesperetin glucuronide, showed a double phase metabolism (Figure 3).

Probably, naringenin and hesperetin were partially cleaved in the first gastro-intestinal tract and rapidly absorbed and metabolized at intestinal/hepatic level, resulting in a first peak approximately $1 \mathrm{~h}$ after capsule ingestion. However, the peak between 5 and $10 \mathrm{~h}$ indicates a more important role of the colonic microbiota in flavanone metabolism [30]. Similarly, ferulic acid glucuronide absorption profile showed a double phase curve (Figure 3). Nevertheless, no ferulic acid was detected in the capsules [23], suggesting that this compound probably originated by catechol-O-methyltransferase (COMT) activity on other hydroxycinnamic acids present in the capsules [39]. 

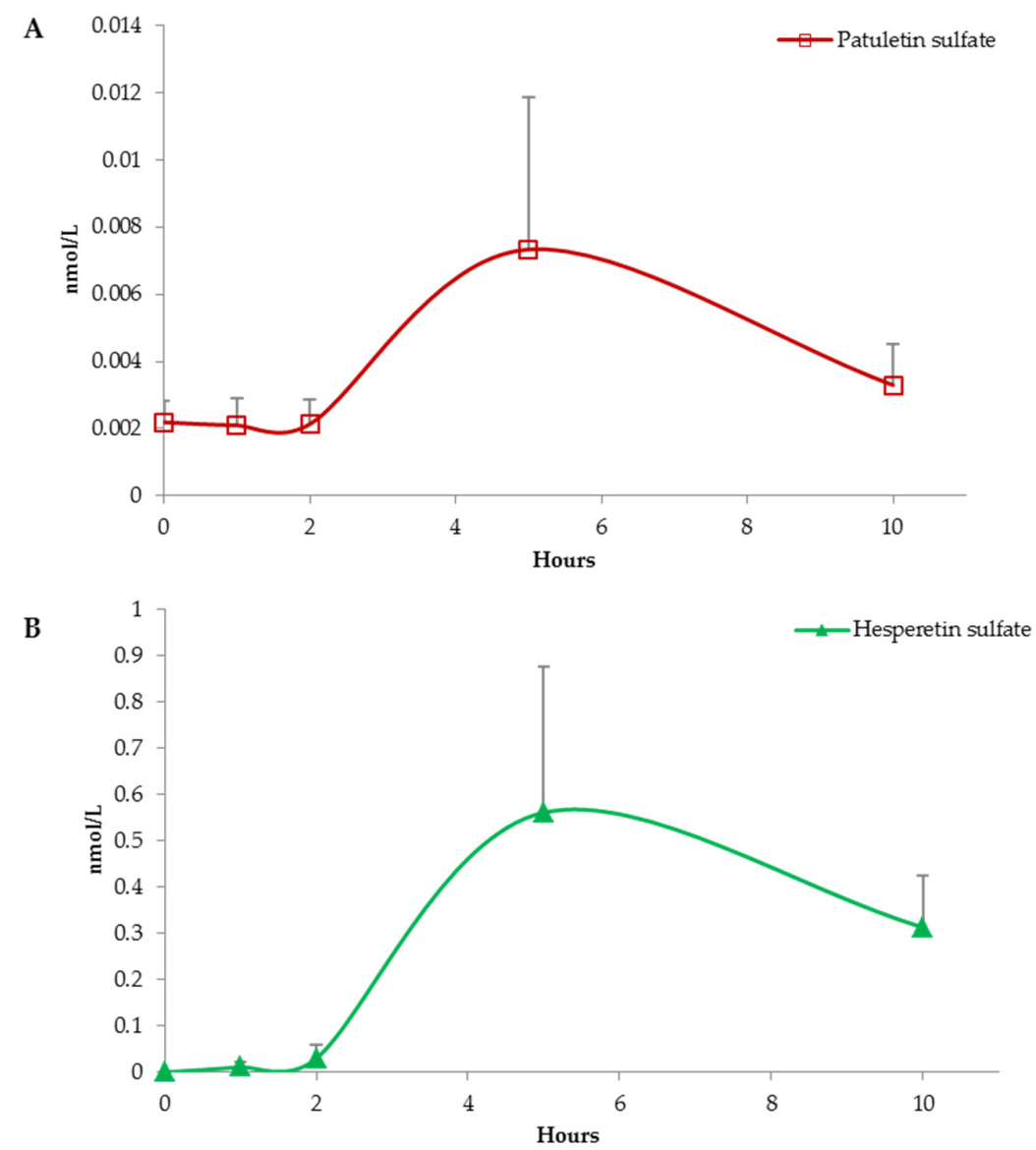

Figure 2. Absorption curves of patuletin sulfate in graph (A) and hesperetin sulfate in graph (B). Data are expressed as mean values and bars represent standard error of means (SEM).

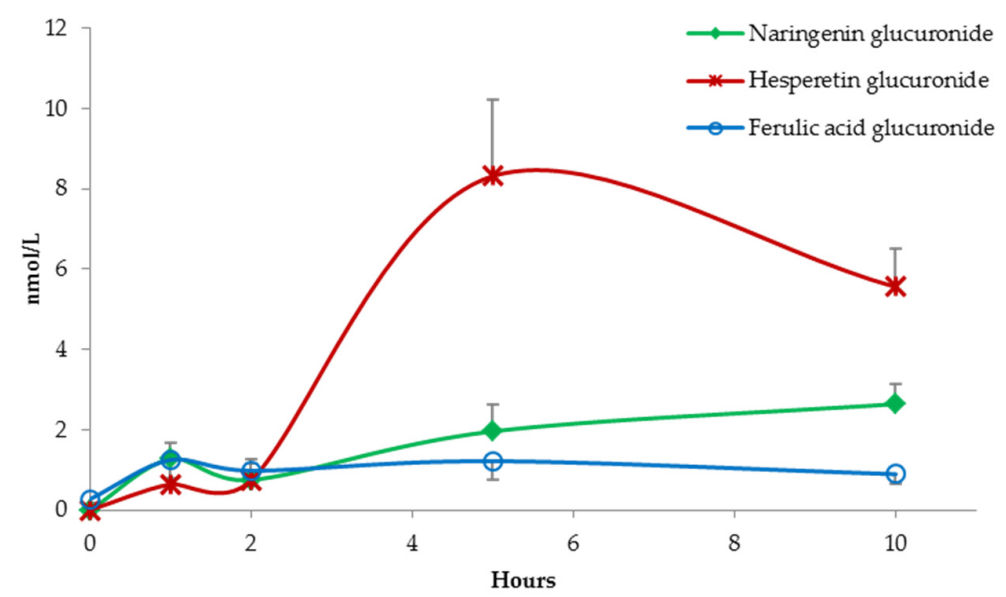

Figure 3. Absorption curves of naringenin glucuronide, hesperetin glucuronide, and ferulic acid glucuronide. Data are expressed as mean values and bars represent standard error of means (SEM).

Finally, most (poly)phenolic compounds passing unmodified and unabsorbed through the first gastro-intestinal tract become a suitable substrate for the locally hosted microbiota. Several modifications on native compounds have been reported to be catalyzed by microbial enzymes, resulting in the formation of low molecular weight compounds [40], which are efficiently absorbed by colonocytes before hepatic conjugation. 
The three phenyl- $\gamma$-valerolactone derivatives, hydroxyphenylpropionic acid, pyrogallol, dihydroxybenzoic acid, and methyl-trihydroxybenzoic acid, having a peak concentration registered within 5 or $10 \mathrm{~h}$, are typical metabolites generated by the host microbiota activity [41-43] (Figure 4).
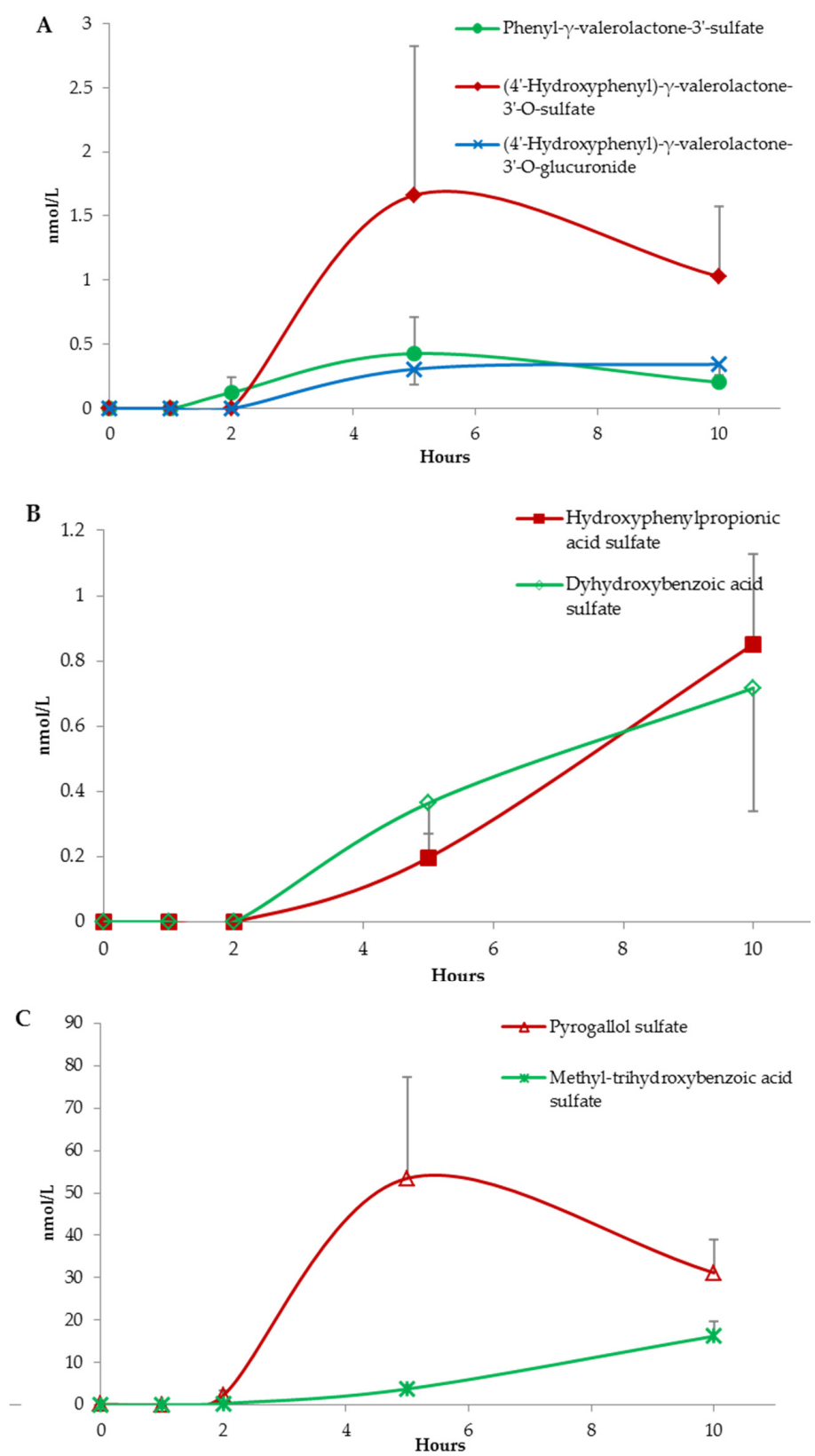

Figure 4. Absorption curves of phenyl- $\gamma$-valerolactone- $3^{\prime}-O$-sulfate, (4'-hydroxyphenyl)- $\gamma$ valerolactone- $3^{\prime}$-O-sulfate, and ( $4^{\prime}$-hydroxyphenyl)- $\gamma$-valerolactone- $3^{\prime}$-O-glucuronide in graph (A); hydroxyphenylpropionic acid sulfate and (dihydroxybenzoic acid sulfate in graph (B); and pyrogallol sulfate and methyl-trihydroxybenzoic acid sulfate in graph $(\mathbf{C})$. Data are expressed as mean values and bars represent standard error of means (SEM).

\section{Discussion}

In the present study, the absorption profile of (poly)phenolic compounds derived from the ingestion of Juice PLUS $+{ }^{\circledR}$ berry, fruit and vegetable blend capsules has been investigated. A total of 20 circulating metabolites have been identified, or tentatively identified, and quantified. As expected, detected metabolites derived from different (poly)phenols and appeared at different 
times in plasma. Flavonol metabolites principally originated in the first part of the gastro-intestinal tract, as previously reported by other authors. Feliciano and colleagues [41] reported a time to reach the maximum plasma concentration $\left(\mathrm{T}_{\max }\right)$ for kaempferol 3-O-glucuronide and quercetin 3-O-glucuronide of about $2 \mathrm{~h}$. An absorption curve similar to those reported in the present study for quercetin 3-glucuronide and quercetin 3-sulfate was reported by Mullen and colleagues, who highlighted a concentration peak between 1 and $2 \mathrm{~h}$ after onion ingestion [44]. To the best of our knowledge, this is the first study in which plasma concentrations of patuletin and myricetin metabolites have been reported, suggesting that other flavonols could be effectively absorbed and hence investigated for their potential bioactivity. Moreover, a new flavone metabolite at plasmatic level has been detected, namely diosmetin sulfate. Its early peak plasma level registered at $1 \mathrm{~h}$ is in contrast with scientific data previously reported for other flavones, like apigenin. The $T_{\max }$ of apigenin (measured as aglycone after enzymatic hydrolysis) has been reported to be around $7 \mathrm{~h}$ after consumption of parsley [45]. The few human feeding studies involving flavones available so far reported a low bioavailability for this phytochemical $[18,46]$, which could be ascribable to the low absorption of flavones, as demonstrated in the present investigation. Conversely, several scientific data are available for naringenin and hesperetin absorption, and all studies agree about the importance of colonic microbiota activity on this class of (poly)phenols [47-49]. The absorption curves observed in the present work confirm that flavanone metabolism principally occurs in the large intestine. Brett and colleagues reported a plasma concentration peak of flavanone conjugates $6 \mathrm{~h}$ after orange consumption [50], whereas the highest naringenin and hesperetin derivative urinary excretion has been reported within 2 and $10 \mathrm{~h}$ after orange juice consumption [30]. Flavan-3-ols were the most representative compounds in berry blend capsules [23]. However, no catechin monomer conjugates nor dimers or oligomeric proanthocyanidins were detected in plasma. However, in vivo studies have shown that both monomers and high molecular weight flavan-3-ols are effectively degraded by the gut microbiota into hydroxyphenyl- $\gamma$-valerolactones [51-53]. Many phenyl- $\gamma$-valerolactone derivatives have been detected after green tea [51,54], cocoa [34,55,56], wine [57] and almond [58] consumption. Partially compensating for the absence of flavan-3-ol monomers in plasma in the present study, three phenyl- $\gamma$-valerolactones were detected and quantified.

It was then demonstrated that phenyl- $\gamma$-valerolactones represent an intermediate step in the microbial metabolism of flavan-3-ols and that other low molecular weight compounds, such as phenylacetic, phenylpropionic, benzoic acids derivatives [59] and hippuric acid, which derive from benzoic acids [60], could be formed. Likewise, flavonols, flavones, and flavanones, could be degraded into smaller phenolics, namely phenylacetic, phenylpropionic, and benzoic acid derivatives [61]. Similarly, anthocyanins undergo an important colonic set of transformations, giving rise to low molecular weight metabolites. After glucosidic cleavage of the sugar moiety, cyanidin could be the precursor of caffeic acid, from which ferulic and isoferulic acids could be formed after COMT activity [62-64]. Ferulic acid may undergo further phase II metabolism, namely sulfation and glucuronidation, generating conjugated ferulic derivatives [39,65]. Feliciano and colleagues recently hypothesized that peonidin could also lead to the formation of ferulic acids [41]. Moreover, anthocyanins could be converted into small phenolics such as phenylpropionic, phenylacetic, benzoic acids, and pyrogallol $[62,64,66]$.

Concerning pyrogallol, the high plasmatic concentration of pyrogallol sulfate between 5 and $10 \mathrm{~h}$ recorded in the present study is in agreement with a $\mathrm{T}_{\max }$ reported after cranberry consumption [41]. Gallic acid, which was present in the capsules, could have contributed to the formation of small metabolites [67], but also to other compounds like methylgallic acid [68], and, after phase II metabolism, to its 3-O-sulfate derivative [69].

It now appears clear that the extensive bioconversion of (poly)phenol compounds strictly depends on the characteristics of individual colonic microbiota, and differences in microbiota composition now allow to discriminate phenotypes associated with producers and non-producers of specific metabolites $[67,70,71]$. As a matter of fact, inter-individual differences in the intestinal ecology may 
lead to differences in bioavailability, linked to specific metabolite production and, ideally, to differences in health benefits [72]. In the present study, the absorption of phenolics and the production of their metabolites is accompanied by a considerable inter-individual variability, plausibly due to the interaction between these compounds and the gut microbiota of the host. A clear example of this large variability among participants concerns the circulating concentration of phenyl- $\gamma$-valerolactones resulting from the catabolic transformations of catechins and procyanidins, operated mainly by Clostridium coccoides and Bifidobacterium spp. [73]. By analyzing the absorption curves of each participants, five subjects resulted as abundant producers of phenyl- $\gamma$-valerolactones, whereas the remaining subjects produced only extremely small amounts of these compounds (data not shown), suggesting marked variations in the colonic microflora of the individual volunteers. The inter-individual variability can affect not only the quantity of metabolites but also the timing of their appearance. For instance, the wide bars observed in the curves of kaempferol glucuronide are ascribable to the fact that these metabolites disappeared after 5 and $10 \mathrm{~h}$ in almost all the participants with the only exception of two subjects, who showed a second, later peak, probably due to colonic absorption. Considering this large variability, there is an increased interest in stratifying future study participants based on their polyphenol-metabolizing phenotypes (i.e., metabotypes) [72], and the present study supports the hypothesis that this variability should be carefully considered as a confounder of in vivo studies evaluating health effects of these phytochemicals. Finally, considering all the circulating detected metabolites, excluding those compounds whose origin could be attributed to endogenous precursors, such as hippuric acid, 4-hydroxyhippuric acid, and feruloylglycine [18,35], a "global" curve could be depicted to summarize the totality of quantified phenolic metabolites at every specific time point (Figure 5). Observing this graph, some considerations can be drawn: (i) the absorption and metabolism of (poly)phenols in the first gastro-intestinal tract ( $1-2 \mathrm{~h}$ after capsule ingestion) is low when compared to what occurs in the colon (5-10 h after capsule consumption); (ii) the plasma curves of the phenolic metabolites clearly highlighted the deep interaction between these compounds and the gut microbiota; (iii) the beneficial effects attributed to the regular consumption of these capsules does not depend on very high concentration of phenolic metabolites circulating after their consumption. In fact, plasmatic metabolites rarely exceeded nanomolar concentrations, as previously reported $[18,19]$. However, the effect of the regular and long time intake of these products may modify the way our organism interacts with the contained phenolics, perhaps improving its ability to absorb some of them at small intestinal level. Moreover, a modulation of the colonic microbiota in the long run seems plausible, perhaps in the direction of improved transformations, leading to increased absorption of microbial metabolites. Finally, the large variability observed in this short acute absorption study should be taken into consideration in future interventions, as not all the recruited volunteers might deal with Juice PLUS $+{ }^{\circledR}$ phenolics in the same way.

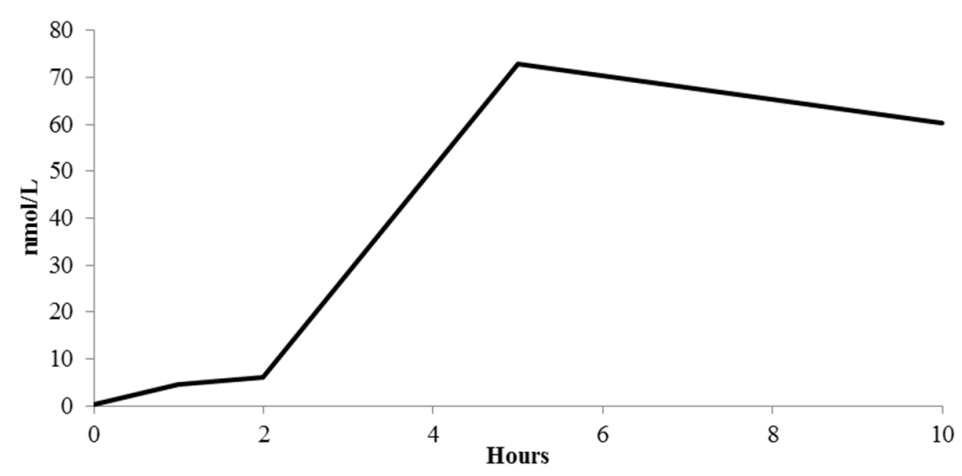

Figure 5. Total concentration of all circulating (poly)phenol metabolites, excluding hippuric acid, 4-hydroxyhippuric acid, and feruloylglycine. Data are expressed as mean values. 


\section{Conclusions}

In conclusion, the consumption of Juice PLUS $+{ }^{\circledR}$ Vineyard, Fruit, Vegetable blend, containing 36 different fruits, vegetables, and berries, allowed the effective absorption of several (poly)phenolic compounds. The capsules therefore ensure potential health-promoting molecules to be potentially available to target tissues and organs, presumably becoming responsible for the previously observed health effects.

Supplementary Materials: The following are available online at http://www.mdpi.com/2072-6643/9/3/194/s1, Table S1: List of forbidden and permitted foods during the (poly)phenol-poor diet. Figure S1: Absorption curves of (a) hippuric acid, (b) 4-hydroxyhippuric acid, (c) feruloylglycine. Data are expressed as mean values and bars represent standard error of means (SEM).

Acknowledgments: Sponsor/Funder is Green Beat-Institute of Nutrient Research and Sports Nutrition, Petersbergenstrasse 95b, 8042 Graz, Austria.

Author Contributions: Manfred Lamprecht and Daniele Del Rio conceived and designed the experiments; Manfred Lamprecht, Daniela-Eugenia Malliga and Sandra Holasek performed the experiments; Daniela-Eugenia Malliga was the study physician, Letizia Bresciani and Giacomo Brigati analyzed the samples and the data; Pedro Mena, Michele Tassotti and Luca Calani contributed with materials and analysis tools; Letizia Bresciani and Daniela Martini wrote the paper; Furio Brighenti critically read and improved the manuscript.

Conflicts of Interest: Manfred Lamprecht is research consultant of The Juice Plus $+{ }^{\circledR}$ Company, NSA LLC.

\section{References}

1. Agudo, A. Measuring intake of fruit and vegetables. In Joint FAO/WHO Workshop on Fruit and Vegetables for Health, WHO/FAO, Kobe, Japan, 1-3 September 2004; World Health Organization: Geneva, Switzerland, 2005.

2. World Health Organization. European Action Plan for Food and Nutrition Policy 2007-2012; World Health Organization: Copenhagen, Denmark, 2008.

3. Boeing, H.; Bechthold, A.; Bub, A.; Ellinger, S.; Haller, D.; Kroke, A.; Leschik-Bonnet, E.; Muller, M.J.; Oberritter, H.; Schulze, M.; et al. Critical review: Vegetables and fruit in the prevention of chronic diseases. Eur. J. Nutr. 2012, 51, 637-663. [CrossRef] [PubMed]

4. Lock, K.; Pomerleau, J.; Causer, L.; Altmann, D.R.; McKee, M. The global burden of disease attributable to low consumption of fruit and vegetables: Implications for the global strategy on diet. Bull. WHO 2005, 83, 100-108. [PubMed]

5. Leenders, M.; Sluijs, I.; Ros, M.M.; Boshuizen, H.C.; Siersema, P.D.; Ferrari, P.; Weikert, C.; Tjonneland, A.; Olsen, A.; Boutron-Ruault, M.C.; et al. Fruit and vegetable consumption and mortality: European prospective investigation into cancer and nutrition. Am. J. Epidemiol. 2013, 178, 590-602. [CrossRef] [PubMed]

6. Boffetta, P.; Couto, E.; Wichmann, J.; Ferrari, P.; Trichopoulos, D.; Bueno-de-Mesquita, H.B.; van Duijnhoven, F.J; Buchner, F.L.; Key, T.; Boeing, H.; et al. Fruit and vegetable intake and overall cancer risk in the European Prospective Investigation into Cancer and Nutrition (EPIC). J. Natl. Cancer Inst. 2010, 102, 529-537. [CrossRef] [PubMed]

7. Bazzano, L.A.; Li, T.Y.; Joshipura, K.J.; Hu, F.B. Intake of fruit, vegetables, and fruit juices and risk of diabetes in women. Diabetes Care 2008, 31, 1311-1317. [CrossRef] [PubMed]

8. Mursu, J.; Virtanen, J.K.; Tuomainen, T.P.; Nurmi, T.; Voutilainen, S. Intake of fruit, berries, and vegetables and risk of type 2 diabetes in Finnish men: The Kuopio Ischaemic Heart Disease Risk Factor Study. Am. J. Clin. Nutr. 2014, 99, 328-333. [CrossRef] [PubMed]

9. Public Health England; Food Standards Agency. Results of the National Diet and Nutrition Survey (NDNS) Rolling Programme for 2008 and 2009 to 2011 and 2012; National Diet and Nutrition Survey (NDNS); Public Health England: London, UK, 2014.

10. Leclercq, C.; Arcella, D.; Piccinelli, R.; Sette, S.; Le Donne, C.; Turrini, A. The Italian National Food Consumption Survey INRAN-SCAI 2005-06: Main results in terms of food consumption. Public Health Nutr. 2009, 12, 2504-2532. [CrossRef] [PubMed]

11. Niva, M. 'All foods affect health': Understandings of functional foods and healthy eating among health-oriented Finns. Appetite 2007, 48, 384-393. [CrossRef] [PubMed]

12. Nile, S.H.; Park, S.W. Edible berries: Bioactive components and their effect on human health. Nutrition 2014, 30, 134-144. [CrossRef] [PubMed] 
13. Murphy, M.M.; Barraj, L.M.; Herman, D.; Bi, X.; Cheatham, R.; Randolph, R.K. Phytonutrient intake by adults in the United States in relation to fruit and vegetable consumption. J. Acad. Nutr. Diet. 2012, 112, 222-229. [CrossRef] [PubMed]

14. Liu, R.H. Dietary bioactive compounds and their health implications. J. Food Sci. 2013, 78 (Suppl. 1), A18-A25. [CrossRef] [PubMed]

15. Grosso, G.; Stepaniak, U.; Topor-Madry, R.; Szafraniec, K.; Pajak, A. Estimated dietary intake and major food sources of polyphenols in the Polish arm of the HAPIEE study. Nutrition 2014, 30, 1398-1403. [CrossRef] [PubMed]

16. Pérez-Jiménez, J.; Fezeu, L.; Touvier, M.; Arnault, N.; Manach, C.; Hercberg, S.; Galan, P.; Scalbert, A. Dietary intake of 337 polyphenols in French adults. Am. J. Clin. Nutr. 2011, 93, 1220-1228. [CrossRef] [PubMed]

17. Tresserra-Rimbau, A.; Medina-Remon, A.; Perez-Jimenez, J.; Martinez-Gonzalez, M.A.; Covas, M.I.; Corella, D.; Salas-Salvado, J.; Gomez-Gracia, E.; Lapetra, J.; Aros, F.; et al. Dietary intake and major food sources of polyphenols in a Spanish population at high cardiovascular risk: The PREDIMED study. Nutr. Metab. Cardiovasc. Dis. 2013, 23, 953-959. [CrossRef] [PubMed]

18. Del Rio, D.; Rodriguez-Mateos, A.; Spencer, J.P.; Tognolini, M.; Borges, G.; Crozier, A. Dietary (poly)phenolics in human health: Structures, bioavailability, and evidence of protective effects against chronic diseases. Antioxid. Redox Signal. 2013, 18, 1818-1892. [CrossRef] [PubMed]

19. Rodriguez-Mateos, A.; Vauzour, D.; Krueger, C.G.; Shanmuganayagam, D.; Reed, J.; Calani, L.; Mena, P.; del Rio, D.; Crozier, A. Bioavailability, bioactivity and impact on health of dietary flavonoids and related compounds: An update. Arch. Toxicol. 2014, 88, 1803-1853. [CrossRef] [PubMed]

20. Grosso, G.; Stepaniak, U.; Micek, A.; Stefler, D.; Bobak, M.; Pajak, A. Dietary polyphenols are inversely associated with metabolic syndrome in Polish adults of the HAPIEE study. Eur. J. Nutr. 2016. [CrossRef] [PubMed]

21. Liu, Y.J.; Zhan, J.; Liu, X.L.; Wang, Y.; Ji, J.; He, Q.Q. Dietary flavonoids intake and risk of type 2 diabetes: A meta-analysis of prospective cohort studies. Clin. Nutr. 2014, 33, 59-63. [CrossRef] [PubMed]

22. Grosso, G.; Godos, J.; Lamuela-Raventos, R.; Ray, S.; Micek, A.; Pajak, A.; Sciacca, S.; D'Orazio, N.; del Rio, D.; Galvano, F. A comprehensive meta-analysis on dietary flavonoid and lignan intake and cancer risk: Level of evidence and limitations. Mol. Nutr. Food Res. 2016. [CrossRef] [PubMed]

23. Bresciani, L.; Calani, L.; Cossu, M.; Mena, P.; Sayegh, M.; Ray, S.; del Rio, D. (Poly)phenolic characterization of three food supplements containing 36 different fruits, vegetables and berries. PharmaNutrition 2015, 3, 11-19. [CrossRef]

24. Lamprecht, M.; Obermayer, G.; Steinbauer, K.; Cvirn, G.; Hofmann, L.; Ledinski, G.; Greilberger, J.F.; Hallstroem, S. Supplementation with a juice powder concentrate and exercise decrease oxidation and inflammation, and improve the microcirculation in obese women: Randomised controlled trial data. Br. J. Nutr. 2013, 110, 1685-1695. [CrossRef] [PubMed]

25. De Spirt, S.; Sies, H.; Tronnier, H.; Heinrich, U. An encapsulated fruit and vegetable juice concentrate increases skin microcirculation in healthy women. Skin Pharmacol. Physiol. 2012, 25, 2-8. [CrossRef] [PubMed]

26. Roll, S.; Nocon, M.; Willich, S.N. Reduction of common cold symptoms by encapsulated juice powder concentrate of fruits and vegetables: A randomised, double-blind, placebo-controlled trial. Br. J. Nutr. 2011, 105, 118-122. [CrossRef] [PubMed]

27. Nantz, M.P.; Rowe, C.A.; Nieves, C., Jr.; Percival, S.S. Immunity and antioxidant capacity in humans is enhanced by consumption of a dried, encapsulated fruit and vegetable juice concentrate. J. Nutr. 2006, 136, 2606-2610. [PubMed]

28. Plotnick, G.D.; Corretti, M.C.; Vogel, R.A.; Hesslink, R., Jr.; Wise, J.A. Effect of supplemental phytonutrients on impairment of the flow-mediated brachial artery vasoactivity after a single high-fat meal. J. Am. Coll. Cardiol. 2003, 41, 1744-1749. [CrossRef]

29. Ottaviani, J.I.; Momma, T.Y.; Heiss, C.; Kwik-Uribe, C.; Schroeter, H.; Keen, C.L. The stereochemical configuration of flavanols influences the level and metabolism of flavanols in humans and their biological activity in vivo. Free Radic. Biol. Med. 2011, 50, 237-244. [CrossRef] [PubMed]

30. Pereira-Caro, G.; Borges, G.; van der Hooft, J.; Clifford, M.N.; Del Rio, D.; Lean, M.E.; Roberts, S.A.; Kellerhals, M.B.; Crozier, A. Orange juice (poly)phenols are highly bioavailable in humans. Am. J. Clin. Nutr. 2014, 100, 1378-1384. [CrossRef] [PubMed] 
31. Curti, C.; Brindani, N.; Battistini, L.; Sartori, A.; Pelosi, G.; Mena, P.; Brighenti, F.; Zanardi, F.; del Rio, D. Catalytic, Enantioselective Vinylogous Mukaiyama Aldol Reaction of Furan-Based Dienoxy Silanes: A Chemodivergent Approach to $\gamma$-Valerolactone Flavan-3-ol Metabolites and $\delta$-Lactone Analogues. Adv. Synth. Catal. 2015. [CrossRef]

32. Liu, Y.; Lien, I.F.; Ruttgaizer, S.; Dove, P.; Taylor, S.D. Synthesis and protection of aryl sulfates using the 2,2,2-trichloroethyl moiety. Org. Lett. 2004, 6, 209-212. [CrossRef] [PubMed]

33. Zhang, M.; Jagdmann, G.E., Jr.; Van Zandt, M.; Sheeler, R.; Beckett, P.; Schroeter, H. Chemical synthesis and characterization of epicatechin glucuronides and sulfates: Bioanalytical standards for epicatechin metabolite identification. J. Nat. Prod. 2013, 76, 157-169. [CrossRef] [PubMed]

34. Urpi-Sarda, M.; Monagas, M.; Khan, N.; Llorach, R.; Lamuela-Raventos, R.M.; Jauregui, O.; Estruch, R.; Izquierdo-Pulido, M.; Andres-Lacueva, C. Targeted metabolic profiling of phenolics in urine and plasma after regular consumption of cocoa by liquid chromatography-tandem mass spectrometry. J. Chromatogr. A 2009, 1216, 7258-7267. [CrossRef] [PubMed]

35. Kern, S.M.; Bennett, R.N.; Mellon, F.A.; Kroon, P.A.; Garcia-Conesa, M.T. Absorption of hydroxycinnamates in humans after high-bran cereal consumption. J. Agric. Food Chem. 2003, 51, 6050-6055. [CrossRef] [PubMed]

36. Bresciani, L.; Scazzina, F.; Leonardi, R.; Dall'Aglio, E.; Newell, M.; Dall'Asta, M.; Melegari, C.; Ray, S.; Brighenti, F.; del Rio, D. Bioavailability and metabolism of phenolic compounds from wholegrain wheat and aleurone-rich wheat bread. Mol. Nutr. Food Res. 2016, 60, 2343-2354. [CrossRef] [PubMed]

37. Roowi, S.; Mullen, W.; Edwards, C.A.; Crozier, A. Yoghurt impacts on the excretion of phenolic acids derived from colonic breakdown of orange juice flavanones in humans. Mol. Nutr. Food Res. 2009, 53 (Suppl. 1), S68-S75. [CrossRef] [PubMed]

38. Mulder, T.P.; Rietveld, A.G.; van Amelsvoort, J.M. Consumption of both black tea and green tea results in an increase in the excretion of hippuric acid into urine. Am. J. Clin. Nutr. 2005, 81 (Suppl. 1), 256S-260S. [PubMed]

39. Del Rio, D.; Stalmach, A.; Calani, L.; Crozier, A. Bioavailability of coffee chlorogenic acids and green tea flavan-3-ols. Nutrients 2010, 2, 820-833. [CrossRef] [PubMed]

40. Ozdal, T.; Sela, D.A.; Xiao, J.; Boyacioglu, D.; Chen, F.; Capanoglu, E. The Reciprocal Interactions between Polyphenols and Gut Microbiota and Effects on Bioaccessibility. Nutrients 2016, 8, 78. [CrossRef] [PubMed]

41. Feliciano, R.P.; Boeres, A.; Massacessi, L.; Istas, G.; Ventura, M.R.; Nunes Dos Santos, C.; Heiss, C.; Rodriguez-Mateos, A. Identification and quantification of novel cranberry-derived plasma and urinary (poly)phenols. Arch. Biochem. Biophys. 2016, 599, 31-41. [CrossRef] [PubMed]

42. Ou, K.; Sarnoski, P.; Schneider, K.R.; Song, K.; Khoo, C.; Gu, L. Microbial catabolism of procyanidins by human gut microbiota. Mol. Nutr. Food Res. 2014, 58, 2196-2205. [CrossRef] [PubMed]

43. Dall'Asta, M.; Calani, L.; Tedeschi, M.; Jechiu, L.; Brighenti, F.; del Rio, D. Identification of microbial metabolites derived from in vitro fecal fermentation of different polyphenolic food sources. Nutrition 2012, 28, 197-203. [CrossRef] [PubMed]

44. Mullen, W.; Edwards, C.A.; Crozier, A. Absorption, excretion and metabolite profiling of methyl-, glucuronyl-, glucosyl- and sulpho-conjugates of quercetin in human plasma and urine after ingestion of onions. Br. J. Nutr. 2006, 96, 107-116. [CrossRef] [PubMed]

45. Meyer, H.; Bolarinwa, A.; Wolfram, G.; Linseisen, J. Bioavailability of apigenin from apiin-rich parsley in humans. Ann. Nutr. Metab. 2006, 50, 167-172. [CrossRef] [PubMed]

46. Tang, D.; Chen, K.; Huang, L.; Li, J. Pharmacokinetic properties and drug interactions of apigenin, a natural flavone. Expert Opin. Drug Metab. Toxicol. 2017, 13, 323-330. [CrossRef] [PubMed]

47. Zeng, X.; Bai, Y.; Peng, W.; Su, W. Identification of Naringin Metabolites in Human Urine and Feces. Eur. J. Drug Metab. Pharmacokinet. 2016. [CrossRef] [PubMed]

48. Pereira-Caro, G.; Ludwig, I.A.; Polyviou, T.; Malkova, D.; Garcia, A.; Moreno-Rojas, J.M.; Crozier, A. Identification of Plasma and Urinary Metabolites and Catabolites Derived from Orange Juice (Poly)phenols: Analysis by High-Performance Liquid Chromatography-High-Resolution Mass Spectrometry. J. Agric. Food Chem. 2016, 64, 5724-5735. [CrossRef] [PubMed]

49. Aschoff, J.K.; Riedl, K.M.; Cooperstone, J.L.; Hogel, J.; Bosy-Westphal, A.; Schwartz, S.J.; Carle, R.; Schweiggert, R.M. Urinary excretion of Citrus flavanones and their major catabolites after consumption of fresh oranges and pasteurized orange juice: A randomized cross-over study. Mol. Nutr. Food Res. 2016, 60, 2602-2610. [CrossRef] [PubMed] 
50. Brett, G.M.; Hollands, W.; Needs, P.W.; Teucher, B.; Dainty, J.R.; Davis, B.D.; Brodbelt, J.S.; Kroon, P.A. Absorption, metabolism and excretion of flavanones from single portions of orange fruit and juice and effects of anthropometric variables and contraceptive pill use on flavanone excretion. Br. J. Nutr. 2009, 101, 664-675. [CrossRef] [PubMed]

51. Del Rio, D.; Calani, L.; Cordero, C.; Salvatore, S.; Pellegrini, N.; Brighenti, F. Bioavailability and catabolism of green tea flavan-3-ols in humans. Nutrition 2010, 26, 1110-1116. [CrossRef] [PubMed]

52. Van der Hooft, J.J.; de Vos, R.C.; Mihaleva, V.; Bino, R.J.; Ridder, L.; de Roo, N.; Jacobs, D.M.; van Duynhoven, J.P.; Vervoort, J. Structural elucidation and quantification of phenolic conjugates present in human urine after tea intake. Anal. Chem. 2012, 84, 7263-7271. [CrossRef] [PubMed]

53. Ottaviani, J.I.; Kwik-Uribe, C.; Keen, C.L.; Schroeter, H. Intake of dietary procyanidins does not contribute to the pool of circulating flavanols in humans. Am. J. Clin. Nutr. 2012, 95, 851-858. [CrossRef] [PubMed]

54. Calani, L.; Del Rio, D.; Luisa Callegari, M.; Morelli, L.; Brighenti, F. Updated bioavailability and 48 h excretion profile of flavan-3-ols from green tea in humans. Int. J. Food Sci. Nutr. 2012, 63, 513-521. [CrossRef] [PubMed]

55. Wiese, S.; Esatbeyoglu, T.; Winterhalter, P.; Kruse, H.P.; Winkler, S.; Bub, A.; Kulling, S.E. Comparative biokinetics and metabolism of pure monomeric, dimeric, and polymeric flavan-3-ols: A randomized cross-over study in humans. Mol. Nutr. Food Res. 2015, 59, 610-621. [CrossRef] [PubMed]

56. Urpi-Sarda, M.; Monagas, M.; Khan, N.; Lamuela-Raventos, R.M.; Santos-Buelga, C.; Sacanella, E.; Castell, M.; Permanyer, J.; Andres-Lacueva, C. Epicatechin, procyanidins, and phenolic microbial metabolites after cocoa intake in humans and rats. Anal. Bioanal. Chem. 2009, 394, 1545-1556. [CrossRef] [PubMed]

57. Appeldoorn, M.M.; Vincken, J.P.; Aura, A.M.; Hollman, P.C.; Gruppen, H. Procyanidin dimers are metabolized by human microbiota with 2-(3,4-dihydroxyphenyl)acetic acid and 5-(3,4-dihydroxyphenyl)gamma-valerolactone as the major metabolites. J. Agric. Food Chem. 2009, 57, 1084-1092. [CrossRef] [PubMed]

58. Bartolome, B.; Monagas, M.; Garrido, I.; Gomez-Cordoves, C.; Martin-Alvarez, P.J.; Lebron-Aguilar, R.; Urpi-Sarda, M.; Llorach, R.; Andres-Lacueva, C. Almond (Prunus dulcis (Mill.) D.A. Webb) polyphenols: From chemical characterization to targeted analysis of phenolic metabolites in humans. Arch. Biochem. Biophys. 2010, 501, 124-133. [CrossRef] [PubMed]

59. Zhang, L.; Wang, Y.; Li, D.; Ho, C.T.; Li, J.; Wan, X. The absorption, distribution, metabolism and excretion of procyanidins. Food Funct. 2016. [CrossRef] [PubMed]

60. Pan, P.; Skaer, C.W.; Stirdivant, S.M.; Young, M.R.; Stoner, G.D.; Lechner, J.F.; Huang, Y.W.; Wang, L.S. Beneficial Regulation of Metabolic Profiles by Black Raspberries in Human Colorectal Cancer Patients. Cancer Prev. Res. (Phila.) 2015, 8, 743-750. [CrossRef] [PubMed]

61. Serra, A.; Macià, A.; Romero, M.-P.; Reguant, J.; Ortega, N.; Motilva, M.-J. Metabolic pathways of the colonic metabolism of flavonoids (flavonols, flavones and flavanones) and phenolic acids. Food Chem. 2012, 130, 383-393. [CrossRef]

62. Ludwig, I.A.; Mena, P.; Calani, L.; Borges, G.; Pereira-Caro, G.; Bresciani, L.; del Rio, D.; Lean, M.E.; Crozier, A. New insights into the bioavailability of red raspberry anthocyanins and ellagitannins. Free Radic. Biol. Med. 2015, 89, 758-769. [CrossRef] [PubMed]

63. De Ferrars, R.M.; Cassidy, A.; Curtis, P.; Kay, C.D. Phenolic metabolites of anthocyanins following a dietary intervention study in post-menopausal women. Mol. Nutr. Food Res. 2014, 58, 490-502. [CrossRef] [PubMed]

64. de Ferrars, R.M.; Czank, C.; Zhang, Q.; Botting, N.P.; Kroon, P.A.; Cassidy, A.; Kay, C.D. The pharmacokinetics of anthocyanins and their metabolites in humans. Br. J. Pharmacol. 2014, 171, 3268-3282. [CrossRef] [PubMed]

65. Stalmach, A.; Mullen, W.; Barron, D.; Uchida, K.; Yokota, T.; Cavin, C.; Steiling, H.; Williamson, G.; Crozier, A. Metabolite profiling of hydroxycinnamate derivatives in plasma and urine after the ingestion of coffee by humans: Identification of biomarkers of coffee consumption. Drug Metab. Dispos. 2009, 37, 1749-1758. [CrossRef] [PubMed]

66. Gonzalez-Barrio, R.; Edwards, C.A.; Crozier, A. Colonic catabolism of ellagitannins, ellagic acid, and raspberry anthocyanins: In vivo and in vitro studies. Drug Metab. Dispos. 2011, 39, 1680-1688. [CrossRef] [PubMed]

67. Selma, M.V.; Espin, J.C.; Tomas-Barberan, F.A. Interaction between phenolics and gut microbiota: Role in human health. J. Agric. Food Chem. 2009, 57, 6485-6501. [CrossRef] [PubMed]

68. Margalef, M.; Pons, Z.; Bravo, F.I.; Muguerza, B.; Arola-Arnal, A. Plasma kinetics and microbial biotransformation of grape seed flavanols in rats. J. Funct. Foods 2015, 12, 478-488. [CrossRef] 
69. Pimpao, R.C.; Dew, T.; Figueira, M.E.; McDougall, G.J.; Stewart, D.; Ferreira, R.B.; Santos, C.N.; Williamson, G. Urinary metabolite profiling identifies novel colonic metabolites and conjugates of phenolics in healthy volunteers. Mol. Nutr. Food Res. 2014, 58, 1414-1425. [CrossRef] [PubMed]

70. Rodriguez-Mateos, A.; Cifuentes-Gomez, T.; Gonzalez-Salvador, I.; Ottaviani, J.I.; Schroeter, H.; Kelm, M.; Heiss, C.; Spencer, J.P. Influence of age on the absorption, metabolism, and excretion of cocoa flavanols in healthy subjects. Mol. Nutr. Food Res. 2015, 59, 1504-1512. [CrossRef] [PubMed]

71. Mennen, L.I.; Sapinho, D.; Ito, H.; Galan, P.; Hercberg, S.; Scalbert, A. Urinary excretion of 13 dietary flavonoids and phenolic acids in free-living healthy subjects - variability and possible use as biomarkers of polyphenol intake. Eur. J. Clin. Nutr. 2008, 62, 519-525. [CrossRef] [PubMed]

72. Tomas-Barberan, F.A.; Selma, M.V.; Espin, J.C. Interactions of gut microbiota with dietary polyphenols and consequences to human health. Curr. Opin. Clin. Nutr. Metab. Care 2016, 19, 471-476. [CrossRef] [PubMed]

73. Marin, L.; Miguelez, E.M.; Villar, C.J.; Lombo, F. Bioavailability of dietary polyphenols and gut microbiota metabolism: Antimicrobial properties. Biomed. Res. Int. 2015, 2015, 905215. [CrossRef] [PubMed]

(C) 2017 by the authors. Licensee MDPI, Basel, Switzerland. This article is an open access article distributed under the terms and conditions of the Creative Commons Attribution (CC BY) license (http:/ / creativecommons.org/licenses/by/4.0/). 